Schock, Kurt (2005), Unarmed Uprisings: People Power Movements in Nondemocracies, Minneapolis MN, University of Minnesota Press.

Sharp, Gene (2003), From Dictatorship to Democracy: A Conceptual Framework for Liberation, Boston MA, The Albert Einstein Institution.
Sharp, Gene (2005), Waging Nonviolent Struggle. Boston MA, Porter Sargent.

Zunes, Stephen (1999), The Origins of People Power in the Philippines, in Zunes, et al, Nonviolent Social Movements: A Geographical Perspective, Oxford Blackwell, pp. 129-57.

\title{
Gütekraft: Grundlage der Arbeit für Freiheit, Gerechtigkeit und Menschlichkeit
}

\author{
Martin Arnold*
}

\begin{abstract}
With the term 'goodness-force' an ideal typical model is presented. It is based on different approaches developed and successfully implemented by three protagonists: the Catholic Hildegard Goss-Mayr, the Hindu Mohandas K. Gandhi, and the atheist Bart de Ligt. The synthetized model is an attempt to describe the common core of the various traditions of nonviolence, namely the conception of how nonviolent action typically works. Three manifestations can be differentiated: goodness-force as a pattern of interaction, a concept for behavior, and a human potential. This concept can be practiced on six levels including steps of escalation. The chief elements of impact are action by committed individuals, 'contagion', and mass non-cooperation built on these two.
\end{abstract}

Keywords: ,Goodness-force', Satyagraha, nonviolence, nonviolent action, civil conflict-management Gütekraft, Satyagraha, Gewaltlosigkeit, gewaltfreie Aktion, zivile Konfliktbearbeitung

\section{Einleitung}

A ls Mohandas K. Gandhi das Kunstwort Satyagraha ${ }^{1}$ prägte, nahm er an, dass die Sache, um die es geht, so alt sei „wie die Menschheit“ (Gandhi 1999, 9: 361). Dennoch sah er sich zu dieser begrifflichen Neuschöpfung veranlasst. Mit ihr überführte er auch eine uralte Praxis in ein elaboriertes Handlungskonzept zum Abbau gesellschaftlicher und politischer Missstände, mit dem er vier Jahrzehnte öffentlich experimentierte.

Satyagraha begann am 11. September 1906 (Gandhi 1999, 34: 87): Zusammen mit Gandhi verpflichteten sich 3.000 Inder im Johannesburger Theater feierlich, die gegen die indische Minderheit gerichtete rassistische Gesetzgebung in Südafrika nicht länger hinzunehmen und für ihre Abschaffung notfalls ihr Leben einzusetzen. ${ }^{2}$ Ihnen gegenüber stand die geballte Macht des Regierungschefs General Jan Christiaan Smuts. Bei ihren vielfältigen Aktionen zivilen Ungehorsams (z.B. Verbrennung von Pässen) hatten die engagierten Inder und Inderinnen in der Folge schwer unter dem Einsatz des staatlichen Zwangsapparats zu leiden: Tausende kamen in Gefängnisse, es gab Tote und Verletzte. Doch nach acht Jahren erwies sich Satyagraha

* Dr. Martin Arnold war bis 2010 Pfarrer der Evangelischen Kirche im Rheinland. Er arbeitet seit 1997 ehrenamtlich am Institut für Friedensarbeit und gewaltfreie Konfliktaustragung sowie seit 1998 in der Arbeitsgruppe Gütekraft. Er ist Trainer für Gewaltfreie Aktion und wirkte vielfach an solchen Aktionen mit. 1997 bis 2005 lehrte er an der Universität Marburg im Studiengang Friedens- und Konfliktforschung.

1 Sprich: Satjagrah (zweite Silbe lang)

2 Vgl. Arnold 2011a. als stärker: Die Gesetze wurden zurückgenommen. ${ }^{3}$ Damit war die Grundlage für weitere Anwendungen dieser Streitkunst geschaffen. Dazu gehörte auch der weltberühmte Salzmarsch in Indien 1930, der das Ende der englischen Kolonialherrschaft einläutete.

Im Englischen gab Gandhi Satyagraha meist als non-violence wieder, was zu den deutschen Bezeichnungen ,Gewaltfreiheit ${ }^{\prime}$ oder ,Gewaltlosigkeit' führte. Bei ,non-violence' handelt es sich aber genau genommen um die Übersetzung eines anderen indischen Begriffs mit Jahrtausende alter Tradition: Ahimsa, Nicht-Gewalt. Mit ihr sah Gandhi Satyagraha sachlich zwar eng verbunden. Das Wort ,non-violence' erwies sich aber dennoch als irreführend. Denn die verneinenden Bezeichnungen vermögen die indische Tradition nicht angemessen abzubilden. Gandhi selbst legte besonderen Wert auf den Aspekt der Kraft. Deshalb sprach er häufiger auch von „love-force“, "truth-force" und „soul-force“. ${ }^{4}$,Gewaltlos" oder ,gewaltfrei" suggeriert in der westlichen Welt jedoch das Gegenteil. Dort lässt es eher an Schwäche denken: an die Verneinung von etwas, das als stark gilt (Gewalt). Gandhis Verständnis verkehrt sich mithin in sein Gegenteil.

Allerdings gab es auch im westlichen Sprachraum Versuche, die Bedeutung von Satyagraha angemessener wiederzugeben: „Festhalten an der Wahrheit“ (Sternstein 2008: 59) ist zwar nicht falsch, bleibt aber ebenfalls missverständlich. Satya be-

3 Vgl. Tendulkar 1961-1963, Vol. 1: 149; Gandhi 1972.

4 Siehe Wörterstatistik in http://wp.martin-arnold.eu/wp-content/ uploads/2012/02/2011-1030.-G\%C3\%BCtekraft-Gesamtstudie_002.pdf, ab S. 520. Stand: 29.3.2013. 
deutet für Gandhi etwas anderes, als das Wort ,Wahrheit‘ ausdrückt. Dies zeigt sich an seiner Aussage, „Wahrheit (satya) bedeutet auch Liebe“ (Gandhi 1999, 34: 93). Die Übertragung ,Gütekraft' erscheint folglich zutreffender.

,Gütekraft' findet im Folgenden zweifache Verwendung: Zum einen fungiert es als spezielle Bezeichnung für Gandhis Ansatz. ${ }^{5}$ Zum anderen dient es als allgemeiner Konzeptname für jene bislang ,gewaltfrei' oder ,gewaltlos' genannte Tradition, die sich teilweise unabhängig von Gandhi entwickelte. ${ }^{6}$ Die Bezeichnung ,Gütekraft' scheint gut geeignet, den gemeinsamen Kern der verschiedenen Varianten einschließlich der ,zivilen Konfliktbearbeitung - insbesondere die typische Wirkungsweise gewaltfreien Vorgehens - auszudrücken.

Ausgehend von den Überlegungen dreier ,Baumeister' unterschiedlicher Ansätze soll ein Idealtypus synthetisiert werden. Dabei leiten folgende Fragen die Rekonstruktion an: Erstens: Wie bzw. warum ist es möglich, Personen oder auch Gruppen, die zur Gewalt entschlossen scheinen, ohne Androhung oder Einsatz von Gewalt wirksam entgegenzutreten? Und zweitens: Wie ist demgemäß gewaltfreies Vorgehen zu konzipieren, damit es auch in Konflikten gegen gewaltbereite Gegner funktionieren kann?

\section{Drei Baumeister und drei Baupläne zur Gütekraft}

Als Ausgangspunkte der Rekonstruktion eines idealtypischen Gütekraftkonzepts dienen die Pläne dreier ,Baumeister': Dazu zählen erstens die österreichische Katholikin Hildegard Goss-Mayr (geb. 1930), die mehrmals für den Nobelpreis vorgeschlagene Ehrenpräsidentin des Internationalen Versöhnungsbunds. Zweitens handelt es sich um den indischen Hindu Mohandas K. Gandhi (1869-1948), genannt Mahatma: die Große Seele. Hinzu kommt drittens der niederländische Atheist Bart de Ligt (1883-1938), den Zeitgenossen als „Gandhi des Westens" (Dungen 1990: 43) würdigten.

Für die Auswahl dieser drei ,Baumeister‘ sprechen zwei Gründe. Zum einen sind ihre Konzepte wenigstens einmal nachweislich mit Erfolg zum Einsatz gekommen:7 Goss-Mayrs Ansatz lag der „Rosenkranz-Revolution“ auf den Philippinen 1986 zugrunde, die die Marcos-Diktatur beendete (Goss-Mayr 1986, Zunes 1999). Die Anwendung von Gandhis Satyagraha führte, wie bereits erwähnt, 1914 in Südafrika zur Rücknahme der gegen die indische Minderheit gerichteten rassistischen Gesetze. ${ }^{8}$ Und die Umsetzung von Bart de Ligts Konzept trug in den Niederlanden 1923 zur gesetzlichen Anerkennung der Kriegsdienstverweigerung aus Gewissensgründen bei (Dungen 1989: xvi). Zum anderen besitzen die drei Personen jeweils

5 Sprachliches zu Satyagraha ausführlich bei Blume 1987: passim und Arnold 2011d: 91-104.

6 Einen detaillierten Vergleich von ,Gewaltfreiheit‘ und ,Gütekraft` bietet Arnold 2013.

7 Die Erfolgszuschreibung ist nicht monokausal gemeint, denn letztlich tragen immer viele Ursachen zu den Ergebnissen bei. Vgl.: Arnold 2011b: 61f., 36ff., 153f., 199.

8 Weil Gandhis Beitrag zur Befreiung Indiens von der Kolonialherrschaft durchaus umstritten ist, wird hier auf den eindeutigen Erfolg in Südafrika verwiesen. unterschiedliche kulturelle Hintergründe und religiöse Auffassungen. Damit wird deutlich: Gütekraft ist weder an einen bestimmten Kulturkreis noch an eine bestimmte religiöse Überzeugung gekoppelt. Wie haben die drei ,Baumeister' sich die Wirkungsweise ihres Ansatzes in der praktischen Konfliktbearbeitung vorgestellt?

\subsection{Hildegard Goss-Mayrs christliches Gütekraft- Konzept}

Nach Hildegard Gross-Mayr ${ }^{9}$ besteht der Anfangsimpuls in der Entdeckung der Gütekraft im Eigenen, als eigene Möglichkeit. Wer sich der eigenen Gütekraftpotenz bewusst sei, werde dazu angeregt, anderen Menschen mit Güte zu begegnen. Mit dieser Haltung verbindet sich das Ziel eines „Lebens in Fülle für

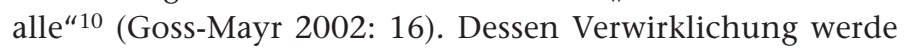
durch Gütekraft - die Kraft der Wahrheit, der Gerechtigkeit und der Liebe - ermöglicht. Sie komme von Gott, die ihr entsprechende Einstellung könne jedoch durch Persönlichkeitsbildung entwickelt und gestärkt werden: „Es kommt darauf an, wie viel Raum man der Liebe in sich gibt und dass man sie in sich wachsen lässt" (Goss-Mayr im Interview mit M.A. am 3.8.2004, unveröffentlicht).

Größere Missstände gütekräftig abzubauen erfordert nach Goss-Mayr Handeln mit Konzept. Demnach muss zur Haltung der Güte Methodenkompetenz hinzukommen, die sich durch Schulung und Erfahrung erwerben lässt. Methodische Hilfsmittel erleichtern die Zusammenarbeit der Engagierten, die Situationsanalyse, den Dialog mit anderen Beteiligten oder auch die selbstkritische Auswertung der Aktivitäten. Das Ziel bestehe darin, die , ansteckende‘ Interaktionsdynamik Gütekraft in Gang zu setzen. Dies geschehe vor allem durch den eigenen Beitrag der Engagierten: Durch konstruktives Handeln sowie durch Nichtzusammenarbeit bauen sie ihren eignen Anteil am Missstand $a b$, indem sie ihn nicht länger dulden oder gar unterstützen. Dieser Beitrag rege andere zur Mitwirkung an, er ,stecke an'. Ein derartiger Effekt lasse sich verbal oder auch durch Aktionen verstärken, die andere Beteiligte in ihrem Gewissen ansprechen. Lassen sich mächtige Schlüsselpersonen dennoch nicht zur Mitwirkung anregen, könne der Einsatz intensiviert werden: Massenhafte Nichtzusammenarbeit gilt als äußerste Aktionsform. Sie könne zum Abbau des Missstands führen, weil Machtausübung auf die Zusammenarbeit von Machtinhabern und Machtunterworfenen angewiesen sei. Unterstützungsentzug führe daher zu Machtverlust, der seinerseits die Erfolgschancen der Engagierten erhöhe. Anhand eines Beispiels: Auf den Philippinen hoben nach Bekanntgabe eines falschen Wahlergebnisses tausende Menschen ihr Geld von Banken ab, die dem Diktator nahestanden. Das Volk stellte sich massenhaft den Panzern entgegen, die die abtrünnigen Militärs töten sollten - und die Panzer stoppten. Marcos' Macht war geschwunden, er verließ das Land. Für diese Zusammenhänge prägten die Philippinen die Ausdrücke,Würde anbieten' und, People Power'.

9 Vgl. Goss-Mayr 1981 und Arnold 2011c.

10 Der Ausdruck ist nach Joh. 10,10 (Jerusalemer Übersetzung) gebildet. 


\subsection{Mohandas K. Gandhis Gütekraft-Konzept Satyagraha}

Mohandas K. Gandhi erklärte Satyagraha ${ }^{11}$ als „die Kraft, die aus Wahrheit und Liebe geboren wird“ (Gandhi 1999, 34: 93). Das Konzept basiert auf der Annahme, jeder Mensch neige aus innerer Verbundenheit mit allen Menschen zu Wohlwollen und Gerechtigkeit. Diese könnten daher bei jedem geweckt und entwickelt werden. Das Wertvollste am Menschen sei sein Potenzial zur Selbstlosigkeit. Das schließe den Einsatz von Satyagraha für eigene Interessen aus.

Dabei lassen sich zwei Bedeutungen unterscheiden: Satyagraha im weiteren Sinne besteht im Einsatz für Wohlwollen und Gerechtigkeit - sei es durch Abbau des eigenen Anteils am Missstand, sei es durch Hilfe oder durch konstruktive Arbeit. Bliebe der gewünschte Erfolg aus, hält Gandhi möglicherweise Satyagraha im engeren Sinne für erforderlich. Dies kann „Satyagraha gegen uns selbst" (Gandhi 1999, 17: 412) sein. Sie wirkt seines Erachtens wie folgt: Die Inkaufnahme eines Verzichts, eines Verlusts oder von Leiden (z.B. durch Fasten) stärke Selbstdisziplin und Selbstlosigkeit sowie die Neigung zu allumfassendem Wohlwollen.

Sollte zur Behebung des Missstands die Mitwirkung anderer Menschen nötig sein, wären diese Personen Gandhi zufolge auf eine Weise anzusprechen, die sie anregt, aus innerer Neigung zu Wohlwollen und Gerechtigkeit zu handeln. Nicht nur die Hoffnung auf diesen gewünschten Effekt, sondern auch die Achtung ihrer Würde verbiete es, die Angesprochenen persönlich oder öffentlich herabzusetzen oder auf andere Weise zu schädigen. Zunächst gelte es jedoch die Frage zu klären, ob die anderen den Missstand überhaupt als solchen erkennen und sich als daran Beteiligte sehen. Sollte dies nicht der Fall sein, müsse die Meinungsdifferenz gewissenhaft bearbeitet werden. Dabei biete es sich an, eine allseits anerkannte Instanz hinzuzuziehen, die z.B. die unklare Faktenlage untersucht. Käme es dabei zu einer Bestätigung sowohl des Missstands als auch der eigenen Beteiligung, dann würden die Betroffenen mitwirken, wenn ihr Wohlwollen und ihre Selbstlosigkeit bereits groß genug seien. Bleibe ihr Engagement weiter aus, so sollen speziell an diese Personen gerichtete Satyagraha-Aktionen im engeren Sinne ihren Gerechtigkeitssinn, ihr Wohlwollen und ihre Selbstlosigkeit ansprechen und stärken. Dies geschieht nach Gandhi, indem die bereits Engagierten ihre eigene Beteiligung am Missstand - etwa den Gehorsam gegenüber einem ungerechten Gesetz - (weiter) abbauen. Dies gelte sogar dann, wenn das für sie mit erheblichen Kosten oder Risiken (z.B. Festnahmen) verbunden wäre. Der Abbau eigener Beteiligung könne auch durch Verzicht auf bestimmte Produkte - etwa Speisen (Gandhi 1977: 276f) - erfolgen, aber auch durch Streik und Boykott als Formen der Nichtzusammenarbeit geschehen. Gerade durch diese Leiden soll der innere Zwiespalt bei den anderen Beteiligten herbeigeführt oder verstärkt werden, der dann den Druck erhöht, dem eigenen Wohlwollen zu folgen und ebenfalls zum Abbau des Missstands beizutragen. Dieser Impuls lasse sich auf indirektem Weg verstärken - etwa durch Öffentlichkeitsarbeit, Gewinnung von Autoritäten oder Anrufung von Gott durch Gebet.
Was aber, wenn Satyagraha bei den Adressaten zur Verhärtung der Position oder gar zur Einleitung von Gegenaktionen führt? Gandhis Antwort lautet: Satyagraha im engeren Sinne ist bis zur Behebung des Missstands weiter zu praktizieren. Die Engagierten sollen darin fortfahren, die eigene Beteiligung am Missstand abzubauen. Indem sie hierbei stärker, als es die bisherigen oder zu erwartenden Gegenaktionen verlangen würden, Furchtlosigkeit, Selbstdisziplin und Leidensbereitschaft gegebenenfalls bis zum Tod zeigen, fordern sie die Angesprochenen solange unüberwindbar heraus, bis auch sie ihren Teil zur Beseitigung des Missstands beitragen.

\subsection{Bart de Ligts Gütekraft-Konzept Geestelijke Weerbaarheid}

Bart de Ligts Ausdruck ,Geestelijke Weerbaarheid' bedeutet geistig-sittliche Streitbarkeit. ${ }^{12}$ „Geistig-sittlich“ verweist auf Potenziale der Menschheit. Das Geistige, die Vernunft, ermögliche es, Wahrheit zu erkennen. Und Sittlichkeit, das moralische Gefühl, führe zu Menschlichkeit. Wahrheit und Menschlichkeit wirken seines Erachtens als „Kräfte, mit denen [...] gerechnet werden muss“ (Ligt 1928, 109). Sie verstärken sich gegenseitig: Beide drängen zum Aufbau menschenwürdiger gesellschaftlicher Verhältnisse in Gerechtigkeit, Freiheit und Menschlichkeit sowie zum Abbau entsprechender Missstände. Dies geschehe sowohl bei Einzelpersonen als auch bei Kollektiven durch Betätigung der eigenen geistig-sittlichen Potenz. Wahrheit und Menschlichkeit bilden die Grundlage sowohl für das Handeln der Engagierten als auch für dessen Wirkung, da sie in der Vernunft und im moralischen Gefühl der anderen Beteiligten Resonanz hervorzurufen vermögen. Arbeit für Freiheit, Gerechtigkeit und Menschlichkeit heißt für de Ligt, selbst frei, gerecht und menschlich zu handeln. Dazu gehören vier Aktivitäten:

a) Bei sich selbst anfangen: Die Grundlage sieht de Ligt in der Bereitschaft zur Selbstverbesserung. Sie beginne mit dem Erkennen zunächst der eigenen geistig-sittlichen Kräfte, gefolgt von der Einsicht in die Möglichkeit, selbst Teil des Missstands bzw. des ihn produzierenden Systems zu sein. Dem schließe sich die Bewusstwerdung der eigenen Anteile an. Dem Erkennen folge das Handeln: Dies beginne damit, eigene Anteile am jeweiligen Missstand abzubauen - etwa durch Verweigerung unwürdiger Arbeit, des Kriegsdienstes oder unmenschlicher Befehle. Die ,Zusammenarbeit mit dem Missstand` werde aufgekündigt. Das Handeln samt der dahinterstehenden inneren Haltung strahle auf andere Menschen aus, bei denen ähnliche Impulse geweckt oder gestärkt würden. Ein Nachahmungseffekt führe dazu, dass auch andere bei sich selbst beginnen. So lasse sich manches Übel beheben. Bei größeren Missständen gehöre jedoch auch Vorbereitung dazu.

b) Sich und andere vorbereiten: Vielfältige Aktivitäten dienen der kurz- oder langfristigen Stärkung der geistig-sittlichen Potenz - und zwar sowohl bei sich selbst, in der eigenen Gemeinschaft, als auch in der breiteren Öffentlichkeit und bei der Gegenseite. Mögliche ,Verstärker‘ werden angesprochen. 
Dazu zählen z.B. angesehene Persönlichkeiten, potenziell sympathisierende Organisationen und Medien. Insgesamt gehe es zum einen um Bewusstseinsarbeit einschließlich Öffentlichkeitsarbeit, aber auch um Wissenschaft und Bildung. Zum anderen bedürfe es organisatorischer Vorbereitungen etwa von Streiks, Boykott, zivilem Ungehorsam oder anderen Massenaktionen. Aber auch auf erwartete Gegenreaktionen gelte es sich entsprechend einzustellen.

c) Mit der gegnerischen Seite kommunizieren: Nach de Ligt gilt es, das Geistig-Sittliche, die moralischen Werte, bei der gegnerischen Seite zunächst zu erkennen, um dann daran anschließend bei ihr entsprechende Impulse zu wecken bzw. zu stärken. Dazu gehöre es, Bande der geistig-sittlichen Sympathie zu knüpfen. Die Engagierten appellieren mit Worten und Taten an Vernunft und sittliches Gefühl. Sie streben an, immer mit der Gegenseite im moralischen Kontakt zu bleiben. Die erwartete Folge: Die gegnerische Seite, bei einem Kollektiv anfangs oft nur ein Teil davon, fühlt sich innerlich derart zur Zusammenarbeit für mehr Freiheit, Gerechtigkeit und Menschlichkeit gedrängt, dass sie am Abbau des Missstands mitwirkt. Sollte sie jedoch zunächst ablehnen oder gar mit Gewalt reagieren, indem sie etwa den staatlichen Unterdrückungsapparat einsetzt, rät de Ligt dazu, die Aktivitäten in starker geistig-sittlicher Haltung fortzusetzen, um den möglicherweise bei Teilen der gegnerischen Seite bereits vorhandenen inneren Drang zu Wahrheit und Menschlichkeit auf eine Weise zu stärken, die z.B. bei Polizisten oder Soldaten zur Befehlsverweigerung führen könne. Infolgedessen nehme die Gegenseite sowohl ihr Eigeninteresse als auch ihre geistig-sittliche Verantwortung neu wahr und sie trage ihrerseits zu mehr Freiheit, Gerechtigkeit und Menschlichkeit sowie zur Überwindung des Missstands bei. Wenn dies noch nicht genügt, hält de Ligt eine weitere Aktivität für angezeigt.

d) Gesellschaftliche Verstärker von Wahrheit und Menschlichkeit mobilisieren: Nun appellieren die Engagierten direkt an die möglichen ,Verstärker', ${ }^{13}$ aber auch an die ,Massen', selbst aktiver zu werden - sei es als vom Missstand Betroffene, sei es aus Solidarität. Die Resonanz in ihrer Vernunft und ihrem sittlichen Gefühl führt nach de Ligts Überzeugung dazu, dass sie das geistig-sittliche Moment und den ursprünglichen Appell der Engagierten weiter verstärken eventuell durch eigene öffentliche Aktivitäten (z.B. Massenveranstaltungen). Dadurch könne der Impuls zu Wahrheit und Menschlichkeit eine Eigendynamik entwickeln. Falle er nicht stark genug aus, so sei der Einsatz so lange fortzusetzen, bis die Zusammenarbeit für Freiheit, Gerechtigkeit und Menschlichkeit den Missstand überwindet.

\section{Idealtypus Gütekraft}

Aus der Darstellung der drei Baupläne wird nun ein gedankliches Modell synthetisiert. Dabei handelt es sich um einen Idealtypus im Sinne Max Webers (Weber 1968: 55). Er beschreibt die typischen Wirkungselemente ,gütekräftigen' (oder

13 Siehe oben. ,gewaltfreien`) Vorgehens. Damit schafft er auch Grundlagen für Anleitungen und Evaluationen ,gewaltfreier Aktionen' besser formuliert: von ,Gütekraft-Aktionen' ${ }^{14}$

\subsection{Zwei Wortbestandteile: ,Güte' und ,Kraft'}

Warum eignet sich ,Gütekraft' in besonderer Weise als Konzeptname? Oder anders gefragt: Welche Inhalte transportiert diese Bezeichnung? Sie vereinbart zwei Wortbestandteile, deren Bedeutung in der Regel nicht zusammengedacht werden, die aber zusammengehören: Güte hat Kraft. Sprachlich leitet sich Güte von ,gut’ ab, das ursprünglich „in ein Gefüge passend" 15 bedeutet. Das Wort besitzt eine intersubjektive und eine subjektive Komponente: Zum einen bezeichnet es eine (intersubjektive) Qualität (z.B. Gütesiegel), zum anderen meint es eine (subjektive) menschliche Einstellung oder Haltung. ${ }^{16}$ Inhaltlich liegt bei beiden Aspekten das Bedeutungsfeld von ,Kraft' nicht fern:

- Güte als Qualität: Bereits die Art und Weise des Handelns, das die gewünschte Verbesserung schon in Gang setzt, lässt erkennen, dass der erstrebte Zustand besser ist als der zu überwindende Zustand. Gandhis den Gegner schonende Vorgehensweise steht nicht nur moralisch höher als Gegner schädigende Vorgehensweisen. Sie verfügt auch durch den Umstand über eine höhere Qualität, dass sie bei geringeren Schäden direkter und oft auch schneller zum Ziel führt. Höhere Qualität besitzt die Kraft, geringere Qualität zu verdrängen, wie bereits der Volksmund weiß: „Das Bessere ist der Feind des Guten".

- Güte als menschliche Haltung: In diesem Sinne äußert sich Güte als Wohlwollen und Gerechtigkeit. Sie zeigt sich im Ziel, eine ,Fülle des Lebens für alle' zu erreichen. Handeln aus dieser Haltung kann durch ,Ansteckung' Kraft nach außen entfalten.

Diese Bedeutungsinhalte des Worts Güte und die Vorstellung damit verbundener Kraft findet in allen drei Einzelkonzepten ihre Entsprechung: Goss-Mayr sieht ,Gütekraft' als ,Kraft der Wahrheit, der Gerechtigkeit und der Liebe'. Gandhi verweist auf die ,Kraft, die aus Wahrheit und Liebe geboren wird'. Und de Ligt spricht von den ,Kräften Wahrheit und Menschlichkeit'. ,Gütekraft' bietet sich also als gemeinsamer Name an, der sowohl die intersubjektiven als auch die subjektiven Bedeutungskomponenten umfasst.

\subsection{Drei Erscheinungsformen}

Gütekraft zeigt sich als Interaktionsmuster, als Begegnungsbzw. Handlungskonzept und als menschliche Potenz.

14 Die folgenden Beschreibungen basieren auf einem detaillierten Vergleich der drei Konzepte und der Ausarbeitung der Gemeinsamkeiten. Aus Raumgründen werden hier weder die einzelnen Arbeitsschritte noch genaue Bezüge zu den drei Konzepten dargelegt, sondern nur die Ergebnisse präsentiert. Ausführlicher in Arnold 2011b, S. 97-113, vgl. auch Arnold 2011b, S. 7 Anm. 1.

15 http://www.duden.de/rechtschreibung/gut Stand: 4.3.2013.

16 http://www.duden.de/rechtschreibung/Guete Stand: 4.3.2013. 
- Interaktionsmuster Gütekraft: Friedrich Glasl (2011) beschreibt die destruktive Abwärtsspirale gewaltsamer Konfliktaustragung. Gütekraft wirkt in die Gegenrichtung: Sie setzt eine ,Engelsspirale' in Gang. ${ }^{17}$ Das Interaktionsmuster Gütekraft zeigt sich dann, wenn bislang nicht engagierte Menschen auf eine wohlwollende und gerechte Ansprache mit ebenfalls wohlwollend-gerechtem (,gütekräftigem') Handeln reagieren. Dies kommt auf der Mikroebene, z.B. in Familien oder unter Freundinnen und Freunden, häufig vor: Egon Spiegel spricht hier von der „Gewaltfreiheit in alltäglichen Beziehungen“ (2008: 129ff.). Je nach Komplexität des Missstands bzw. der Konfliktlage sind jedoch auch Kompetenz und Beharrlichkeit ${ }^{18}$ nötig.

- Begegnungs- oder Handlungskonzept Gütekraft: Gütekräftiges Handeln wird durch Wohlwollen und Gerechtigkeitssinn geprägt. Familie und Freundschaften wie auch Kulturen und Religionen formen und tradieren entsprechende Einstellungen. Die Haltung der Güte kann unbewusst vorhanden sein oder durch Persönlichkeitsbildung und Methodenkompetenz bewusst entwickelt und eingeübt werden. Wo Güte in Verbindung mit Kompetenz im Zusammentreffen mit anderen Menschen oder im Abbau bestehender Missstände praktisch wird, liegt das Begegnungs- und Handlungskonzept Gütekraft vor. Alle drei Konzept-Baumeister unterscheiden zwischen Personen, die sich für die Verbesserung der Verhältnisse durch den Abbau eines Missstands engagieren, und anderen, die dies noch nicht tun. Zu letzteren gehören zu Beginn der Aktivitäten auch die Hauptverantwortlichen oder Verursacher des Missstands, die also möglicherweise Gegner in der Sache sind. Sie werden wie andere mehr oder weniger Beteiligte als potenzielle Verbündete angesprochen. Die Anwendung eines gütekräftigen Begegnungs- und Handlungskonzepts erhöht die Wahrscheinlichkeit, das Interaktionsmuster der Gütekraft freizusetzen.

- Gütekraftpotenz: Der Mensch vermag vom frühen Kindesalter an spontan wohlwollend und gerecht zu handeln. ${ }^{19}$ Diese ansprechbare und aktivierbare Fähigkeit stellt die anthropologische Grundannahme des Gütekraftkonzepts dar. Sie lautet: ,Alle Menschen - auch Konfliktgegner - möchten wohlwollend und gerecht behandelt werden; sie neigen nicht nur bewusst oder unbewusst zu Wohlwollen und Gerechtigkeit, sondern sind auch zu einem entsprechenden Handeln prinzipiell in der Lage.' Durch die Entdeckung der Gütekraftpotenz im Eigenen vermögen wir sowohl unser Selbstbild als auch das Bild, das wir uns von anderen Menschen machen, zu verbessern. ${ }^{20}$ Wir können sie dann bewusst verwirklichen und auf sie - zwar ohne Erfolgsgarantie, aber mit guten Gründen - vertrauen.

17 Bereits bei Dieter Senghaas und Johan Galtung finden sich ähnliche Wortschöpfungen, wenn sie - in anderen Kontexten - dem ,Teufelskreis einen ,Engelskreis‘ oder ,Tugendkreis‘ gegenüberstellen (zit. nach Brücher 2011: 312).

18 Dieser Gedanke findet sich in der lateinamerikanischen Bezeichnung für gütekräftiges Vorgehen: firmeza permanente, dauerhafte Festigkeit.

19 Vgl. Tomasello 2010.

20 Vgl. „beziehungszentrisches Selbstbild“ in Arnold 2011b: 164-193.

\subsection{Sechs Entfaltungsstufen}

Das Gütekraftkonzept hat sich in unterschiedlichen Konfliktlagen bewährt. ${ }^{21}$ Dementsprechend lassen sich unterschiedliche Entfaltungsstufen identifizieren. Für die jeweils nächsten bleiben alle vorherigen wichtig. Keine wird übersprungen. Allenfalls lassen sich mehrere gleichzeitig nehmen.

1. Sich selbst erkennen und risikobereit handeln: Gütekräftiges Handeln bedeutet zuerst, sich nach eigenen Anteilen am Missstand zu befragen und diese abzubauen oder konstruktiv zu handeln. Dies schließt die Bereitschaft ein, Kosten oder Risiken auf sich zu nehmen. Oft gelingt es bereits auf dieser ersten Stufe - eventuell auch durch unaufgeforderte Mitwirkung anderer Personen - Missstände abzutragen. Wenn nicht, geht es auf der zweiten Stufe weiter.

2. Aktivitäten auswerten und eigene Handlungsmöglichkeiten verbessern: Dies geschieht etwa durch Ausbildung, einschließlich Persönlichkeitsbildung. Der Abbau des Missstands kann die Mitwirkung weiterer Personen und damit Aktivitäten der dritten Stufe erfordern.

3. Mit anderen gemeinsam den Missstand abbauen: Alle irgendwie Beteiligten werden als potenzielle Verbündete angesprochen. Daher gilt es, abwertende Äußerungen oder andere Schädigungen zu vermeiden (,Gewaltfreiheit'). Erkennen die Angesprochenen die Lage nicht als Missstand oder sehen sie sich an ihm nicht beteiligt, bedarf diese Frage der Klärung. In Respekt der jeweiligen Person werden im Dialog gegebenenfalls Folgen destruktiver Handlungen verdeutlicht und Kritik geübt. Genügen solches Ansprechen und gemeinsame Aktionen nicht zur Behebung des Missstands, beginnt mit der vierten Stufe die gütekräftige Eskalation.

4. Verbreiterung und Intensivierung des Einsatzes (Eskalation I): Appelle oder auch dramatisierende Aktionen sollen die Angesprochenen zur Unterstützung bewegen. Auf diese Weise wird der Dialog gegebenenfalls in der Öffentlichkeit fortgesetzt. Wohlwollend-gerechtes Streiten regt andere, noch nicht Engagierte zum Handeln an (,Mitschwingen'). Je mehr Personen sich beteiligen, desto stärker dürfte sich bei den vorrangig Verantwortlichen der innere Druck erhöhen, zur Behebung des Missstands beizutragen. Wenn sich diese Gruppe jedoch nicht nur gegen die erforderlichen Veränderungen sperrt, sondern auch massiv gegen die Engagierten oder deren Aktivitäten vorgeht, setzt sich der Einsatz auf der nächsten Eskalationsstufe fort.

5. Ausdauer, Vorbereitung und Erhöhung des Einsatzes (Eskalation II): Persönlichkeitsbildende und methodische Vorbereitung hilft dabei, unter erschwerten Bedingungen, d.h. auch bei Schmerzen und Schädigungen, Wohlwollen und Dialogbereitschaft aufrechtzuerhalten. Wenn die vorrangig Verantwortlichen zu extrem schädigenden Gegenmaßnahmen greifen, zeigen die Engagierten durch freiwillige und gut vorbereitete Gütekraft-Aktionen offensiv, dass sie sich nicht einschüchtern lassen und einen hohen Preis zu zahlen bereit sind. So werden die vorrangig Verantwortlichen in ihrem Drang zu Wahrheit und Menschlichkeit intensiv

21 Vgl. „Gütekraftberichte“ in www.guetekraft.net Stand: 1.3.2013. 
angesprochen. Dies soll und kann dazu führen, dass einzelne Personen oder auch Gruppen den Missstand nicht weiter stützen wollen - beispielsweise könnten sich sogenannte Ordnungskräfte ganz oder teilweise weigern, gegen die Engagierten vorzugehen. Falls Schlüsselpersonen auch nach weiteren Gütekraft-Aktionen unzugänglich bleiben und den Abbau des Missstands blockieren, gehen die Aktiven zur letzten Eskalationsstufe über.

6. Massenhafte Nicht-Zusammenarbeit (Eskalation III): Diese Stufe basiert auf der Annahme, dass Unrechtssysteme oder auch einzelne Missstände nur dann überdauern, wenn Menschen sie stützen. Nichtzusammenarbeit hat zum Ziel, dem Missstand bzw. dem System die Unterstützung zu entziehen. Dazu zählen z.B. Ämterrückgabe, Boykott, Streik bzw. Generalstreik, massenhafte Befehls-, Kriegsdienst- und Steuerverweigerung. Hinzu kommen konstruktive Maßnahmen wie etwa die Besetzung und Umwidmung von Gebäuden und Einrichtungen oder der Entwurf einer neuen Verfassung. Nichtzusammenarbeit kann gesteigert und die Macht von Schlüsselpersonen damit so weit untergraben werden, dass sie entweder einlenken oder ihre Macht aufgeben. Allerdings birgt diese Stufe auch Risiken für gütekräftiges Handeln: Sympathisierende, aber auch Agents Provocateurs könnten zur Gewalt greifen und so die Bewegung schwächen. Deshalb achten die gütekräftig Engagierten stets darauf, den Appellcharakter und die Dialogbereitschaft in den Vordergrund zu stellen, damit sich das Handlungsmotiv des Machtentzugs nicht verselbständigt.

\subsection{Drei Hauptwirkungselemente}

Die in den sechs Stufen erkennbaren Wirkungselemente lassen sich auf drei wesentliche zurückführen: Eigentätigkeit, ,Ansteckung' und darauf aufbauende massenhafte Nichtzusammenarbeit.

- Eigentätigkeit: Gütekraft erfasst die ganze Person, die sich entsprechend verändert bzw. weiterentwickelt. Sie animiert dazu, eigenes Handeln in diesem Sinne zu gestalten. Wer gütekräftig agiert, setzt mit den verwendeten Methoden bereits Verbesserungen in Richtung auf das jeweilige Ziel in Gang. Das bekannte Handlungsmotto ,Der Weg ist das Ziel' wird ergänzt durch den umgekehrten Leitspruch ,Das Ziel ist der Weg'.

- ,Ansteckung': Da es sich hier weder um physiologische noch psychologische Erklärungsversuche handelt, dienen im Folgenden Bezeichnungen wie ,Ansteckung' - oder auch ,Mitschwingen' und ,Kraft' - eher als Metaphern. Demnach sprechen die Engagierten andere Menschen durch gütekräftiges Handeln auf eine Weise an, die deren eigene, vielleicht kaum bewusste Neigung zu Wohlwollen und Gerechtigkeit so mitschwingen lässt, dass sie sich ebenfalls zu einem derartigen Handlungsmodus verstehen.

- Nichtzusammenarbeit: Die dritte Wirkungsweise der Nichtzusammenarbeit schließt an die zuvor genannten Komponenten Eigentätigkeit und ,Ansteckung' an: Wenn sich mächtige Schlüsselpersonen trotz starken öffentlichen
Drucks weigern, am Abbau des Missstands mitzuwirken, regen die Engagierten immer mehr Menschen zu organisiertem, gegebenenfalls massenhaftem ,Mitschwingen' in Form von Nichtzusammenarbeit an, sodass die Macht untergraben und die Aufrechterhaltung des Missstands unmöglich wird.

\section{Fazit}

Die vornehmlich empirischen und quantitativen Studien von Schock (2005), Chenoweth \& Stephan (2011) und Nepstad (2011) haben gezeigt: Erfolge gewaltfreien Handelns hängen nicht von der Gewaltbereitschaft der Gegner ab. Dieser Befund wirft die Frage nach den Erfolgsfaktoren auf. Als besonders wichtig gelten in der Literatur gemeinhin: Einheit der Bewegung, Vielfalt der Aktionsformen, hohe Zahl der Aktiven, Nichtschädigung von Personen, Gewinnen von Polizei bzw. Militär (Chenoweth \& Stephan 2011: 50f., 58,195f. sowie Nepstad 2011: 136). Die Untersuchungen zur Gütekraft bieten hierzu einen anthropologisch fundierten Erklärungsansatz. Dieser integriert auch beide Seiten der bislang gängigen Unterscheidung in eine ,prinzipielle', ,ethische', ,spirituelle Gewaltfreiheit und eine ,pragmatische', ,strategische' Gewaltfreiheit. ${ }^{22}$ Diese unfruchtbare, wenn nicht gar kontraproduktive Dichotomisierung erweist sich als überflüssig, da der Gütekraft-Ansatz den Fokus auf den Kraftaspekt sowie die Wirkungsweise des Vorgehens richtet. Darüber hinaus erscheinen Bezeichnungen wie ,gewaltfreier Widerstand' dem Konzept aus mehreren Gründen nicht angemessen: Erstens dient gütekräftiges Vorgehen auch dem Abbau von Missständen ohne konkreten Gegner, dem mit Widerstand begegnet werden könnte. Zweitens widerspricht Widerstand gegen Personen ohnehin dem Konzept. Drittens schließt der Ansatz der Gütekraft einen Missbrauch zu rein egoistischen Zwecken (auch von Kollektiven) zwar nicht aus, er schiebt ihm aber einen stärkeren Riegel vor als einige Konzepte der gewaltfreien Aktion. ${ }^{23}$ Und last but not least nimmt die konstruktive Arbeit einen zentralen Stellenwert ein. Um mit Birgit Berg zu sprechen: „Die überzeugendste Form des NEIN zum Unzumutbaren ist das JA zu den reiferen Möglichkeiten. “24

\section{Literatur}

Arnold, Martin (2011a): Nine-eleven 1906 »Der Beginn von Satjāgrah« - historisch wichtiger als 9/11 2001. http://www.guetekraft.net/ffone/ffLibrary/downloads/dlfiles/2011-0709\%20nineeleven1906.pdf - Stand: 21.2.2013.

Arnold, Martin (2011b): Gütekraft. Ein Wirkungsmodell aktiver Gewaltfreiheit nach Hildegard Goss-Mayr, Mohandas K. Gandhi und Bart de Ligt. Mit einem Geleitwort von Johan Galtung. Baden-Baden: Nomos.

Arnold, Martin (2011c): Gütekraft - Hildegard Goss-Mayrs christliche Gewaltfreiheit. Overath: Bücken \& Sulzer.

\footnotetext{
22 Vgl. z.B. Burrowes 1996: 100.

23 Z. B. das Konzept von Gene Sharp (1973)

24 Plakat der "Wortwerkstatt Poesie und Politik“.
} 
Arnold, Martin (2011d): Gütekraft - Gandhis Satyagraha. Overath: Bücken \& Sulzer.

Arnold, Martin (2011e): Gütekraft - Bart de Ligts humanistische Geestelijke Weerbaarheid. Overath: Bücken \& Sulzer.

Arnold, Martin (2013): Von der Gewaltfreiheit zur Gütekraft. http://www.martin-arnold.eu/wp-content/uploads/2013/05/2013_Gewaltfreiheit_Guetekraft.pdf.

Blume, Michael (1987): Satyagraha. Wahrheit und Gewaltfreiheit, Yoga und Widerstand bei M.K. Gandhi. Gladenbach: Hinder + Deelmann.

Brücher, Gertrud (2011): Gewaltspiralen. Zur Theorie der Eskalation. Wiesbaden: VS-Verlag.

Burrowes, Robert (1996): The strategy of nonviolent defense. A Gandhian approach. Albany (US): State University of New York Press.

Chenoweth, Erica; Stephan, Maria J. (2011): Why Civil Resistance Works. The Strategic Logic of Nonviolent Conflict. New York: Colombia University Press. Kurzfassung online: http:// belfercenter.ksg.harvard.edu/files/IS3301_pp007-044_Stephan_Chenoweth.pdf.

Dungen, Peter van den (1989): Introduction to the 1989 Edition. In: Ligt, Bart de: The Conquest of Violence. An Essay on War and Revolution. London: Pluto Press (Libertarian critique), S. ix-xxvii.

Dungen, Peter van den (1990): Bart de Ligt, Aldous Huxley and the Conquest of Violence. Notes on the publication of a peace classic and an appreciation of De Ligt in Great Britain and the United States of America. In: Dungen, Peter van den; Noordegraaf, Herman; Robben, Wim (Hg.): Bart de Ligt (18831938) Peace Activist and Peace Researcher. Zwolle, NL: Bart de Ligt-Fonds; Stichting Voorlichting Aktieve Geweldloosheid, S. 27-47.

Gandhi, Mohandas K. (1972): Satyagraha in South Africa. Ahmedabad: Navajivan Publishing House.

Gandhi, Mohandas Karamchand (1977): Eine Autobiographie oder Die Geschichte meiner Experimente mit der Wahrheit. Gladenbach: Hinder + Deelmann.

Gandhi, Mahatma (1999): The Collected Works of Mahatma Gandhi (Electronic Book). (CD-ROM, vgl. www.gandhiserve. org). Publications Division (Hg.). New Delhi: Icon Softec.

Glasl, Friedrich (2011): Konfliktmanagement. Ein Handbuch für Führungskräfte, Beraterinnen und Berater. Bern: Haupt.

Goss-Mayr, Hildegard (1981): Der Mensch vor dem Unrecht. Spiritualität und Praxis gewaltloser Befreiung. Wien [u.a.]: Europaverlag.

Goss-Mayr, Hildegard (1986): AKKAPKA - die gewaltfreie Bewegung auf den Philippinen. Dokumente, zusammengestellt von Hildegard Goss-Mayr. In: gewaltfreie aktion, Jg. 17-18, H. 65/66/67 3. u. 4. Quartal 1985, 1. Quartal 1986, S. 28-31.

Goss-Mayr, Hildegard (2002): Elemente der Gütekraft. Anhand von Beispielen erklärt. In: gewaltfreie aktion, Jg. 34, H. 131, S. $16-25$.
Jochheim, Gernot (1977): Antimilitaristische Aktionstheorie, soziale Revolution und soziale Verteidigung. Zur Entwicklung der Gewaltfreiheitstheorie in der europäischen antimilitaristischen und sozialistischen Bewegung 1890-1940, unter besonderer Berücksichtigung der Niederlande. Frankfurt am Main / Assen /Amsterdam: Haag und Herchen /Van Gorcum.

Ligt, Bart de (1925): Kerk, cultuur en samenleving. Tien jaren strijd. Arnheim: Van Loghum Slaterus en Visser.

Ligt, Barthélemy de (1928): Contre la guerre nouvelle. Paris: Rivière.

Ligt, Bart de: The Conquest of Violence (1989). An Essay on War and Revolution. London: Pluto Press (Libertarian critique) (1. Aufl. 1937).

Nepstad, Sharon Erickson (2011): Nonviolent Revolutions. Civil Resistance in the Late 20th Century. Oxford: Oxford University Press.

Schock, Kurt (2005): Unarmed Insurrections. People Power Movements in Nondemocracies. Minneapolis: Univ. of Minnesota Press.

Sharp, Gene (1973): The Politics of Nonviolent Action. 3 Bände. Boston: Porter Sargent.

Spiegel, Egon (2008): Ohne Gewalt leben. Spiritualität und Praxis gewaltfreier Weltgestaltung. In: Nagler, Michael; Spiegel, Egon (Hg.): Politik ohne Gewalt. Prinzipien, Praxis und Perspektiven der Gewaltfreiheit. Berlin: Lit (Friedenswissenschaft, 1), S. 55-136.

Sternstein, Wolfgang (2008): Gandhi und der Westen - eine Geschichte der Missverständnisse. In: Wissenschaft und Frieden 1/2008, 59-62.

Tendulkar, D. G. (1961-1963): Mahatma. Life of Mohandas Karamchand Gandhi. 8 Bände. Bombay: Vithalbhai K. Jhaveri \& D.G. Tendulkar.

Tomasello, Michael (2010): Warum wir kooperieren. Berlin: Suhrkamp.

Weber, Max (1968): Methodologische Schriften. Studienausgabe. Mit einer Einführung besorgt von Johannes Winckelmann. Frankfurt am Main: S. Fischer.

Zunes, Stephen (1999): The Origins of the People Power in the Philippines. In: Zunes, Stephen; Lurtz, Lester R.; Asher, Sarah Beth (Hg.): Nonviolent social movements. A geographical perspective. Malden, Oxford: Blackwell Publishers, S. 129-157. 